\title{
Testing the S-R link hypothesis of P3b: The oddball effect on S1-evoked P3 gets reduced by increased task relevance of S2
}

\author{
Rolf Verleger $^{\mathrm{a}, \mathrm{c}, *}$, Lin Marlena Hamann ${ }^{\mathrm{a}}$, Dariusz Asanowicz ${ }^{\mathrm{a}, \mathrm{b}}$, Kamila Śmigasiewicz ${ }^{\mathrm{a}}$ \\ a Department of Neurology, University of Lübeck, Germany \\ ${ }^{\mathrm{b}}$ Institute of Psychology, Jagiellonian University, Kraków, Poland \\ c Institute of Psychology II, University of Lübeck, Germany
}

\section{A R T I C L E I N F O}

\section{Article history:}

Received 8 September 2014

Accepted 23 February 2015

Available online 20 March 2015

\section{Keywords:}

P300

P3b

Oddball

Response selection

\begin{abstract}
A B S T R A C T
We had previously reported that the oddball effect on the P3b EEG potential evoked by infrequent vs. frequent S1 presented in a sequence of two stimuli, S1 and S2, gets reduced in a "combination task". In this task, responses were determined by the combinations of S1 and S2 rather than by S1 only. We had attributed this reduction of the oddball effect to increased task difficulty. The present study investigated possible reasons for this reduction of $\mathrm{S} 1$-evoked $\mathrm{P} 3 \mathrm{~b}$ in more detail, by making the combination task easier in several respects: allowing for forming associations from $S 1$ to responses (Experiment 1 ), reducing the complexity of stimulus-response (S-R) mappings (Experiment 2), and decreasing S2 relevance in defining responses (Experiment 3). The results showed that only S2 relevance affected the oddball effect on S1evoked P3b. Namely, when S2 attained some relevance by inducing a go/no-go decision for S1-defined responses, the oddball effect on S1-evoked P3b was intermediate between the large effect in the simple oddball task and the small effect in the combination task. The results may be explained in terms of the $\mathrm{S}-\mathrm{R}$ link hypothesis of $\mathrm{P} 3 \mathrm{~b}$ which interprets $\mathrm{P} 3 \mathrm{~b}$ as reflecting reactivation of well-established S-R links.
\end{abstract}

(c) 2015 Elsevier B.V. All rights reserved.

\section{Introduction}

Several lines of evidence have converged to show that the P3b component of the human event-related EEG potential lies at the interface between stimulus $(\mathrm{S})$ processing and response (R) preparation (Gerson, Parra, \& Sajda, 2005; Kelly \& O'Connell, 2013; Connell, Dockree, \& Kelly, 2012; Ouyang, Herzmann, Zhou, \& Sommer, 2011; Poli, Cinel, Citi, \& Sepulveda, 2010; Saville et al., 2011; Verleger, Jaśkowski, \& Wascher, 2005; Verleger, Schroll, \& Hamker, 2013). In this context, Verleger, Metzner, Ouyang, Śmigasiewicz, and Zhou (2014) have recently specified what might be P3b's function, referring to the well-established "oddball" effect. The oddball effect is the massive increase of P3b amplitudes with infrequent stimuli when two stimuli, one rare and one frequent, are presented in unpredictable random series and require different responses (Duncan-Johnson \& Donchin, 1977; Johnson \& Donchin, 1980; Ritter \& Vaughan, 1969; Squires, Squires, \& Hillyard, 1975). Verleger, Metzner, et al. (2014) suggested that P3b in general, and the oddball P3 in particular, reflects reactivation of some already

\footnotetext{
* Corresponding author at: Klinik für Neurologie, Universität Lübeck, D 23538 Lübeck, Germany. Tel.: +49 451 5002916; fax: +49 4515002489.

E-mail address: rolf.verleger@neuro.uni-luebeck.de (R. Verleger).
}

well-established S-R link that is currently not in an activated state. They argued that in most standard laboratory task (typically consisting of long series of trials) there is no particular selection of new responses in each successive trial. Rather, a few fixed S-R links are established by instruction and practice, most frequently only two (e.g., "frequent $S \rightarrow$ left key", "rare $S \rightarrow$ right key"). If during some consecutive trials only one of these S-R links is used, the other one, not having been used for some time, will have to be reactivated when the corresponding stimulus is perceived. This process of reactivating well-established S-R-links is assumed to be reflected in P3b amplitude.

This hypothesis was put to test by Verleger, Baur, Metzner, and Śmigasiewicz (2014). In their modification of the oddball task, two stimuli were presented (S1 and S2) and responses were defined either by only one of these stimuli or by their combination. S1 was the letter X or U, one frequent and one rare ( $80 \%$ vs. $20 \%)$. The letter was accompanied or followed (as will be the case in the present study) by a blue or yellow frame (S2) that surrounded letter position. In the standard oddball condition, key selection depended on the S1 letter only and S2 served just as "go" signal for pressing that key, S2 color being irrelevant. In this easy task, a large oddball effect was obtained on the S1-evoked P3 amplitudes. In contrast, in the "combination task", S2 color determined the key to be pressed depending on the letter, e.g., blue frame meant "left" 
and yellow frame "right" after frequent X, but blue meant "right" and yellow "left" after infrequent U. In this task, the oddball-P3 evoked by S1 (in this example by infrequent Us) was massively reduced. When conceiving of $\mathrm{P} 3 \mathrm{~b}$ being independent of response processing ("stimulus evaluation hypothesis", e.g., Callaway, 1983; Duncan et al., 2009) difficulty of response selection is not expected to modify the oddball effect because in any case the S1 letters are easily identified and are task-relevant. When, on the other hand, P3b is assumed to reflect decision processes (O'Connell, Dockree, \& Kelly, 2012; Kelly \& O'Connell, 2013) then, if anything, P3b is expected to increase when difficult decisions have to be made. In contrast, Verleger, Metzner, et al.'s (2014) conception of P3b as reflecting reactivation of readily available S-R links may account for this reduction of $\mathrm{P} 3 \mathrm{~b}$ by assuming that such links were not readily available with the infrequent stimuli in the combination task.

The question still remains what actually are the factors responsible for unavailability of S-R links in this combination task. Several possibilities will be tested in the present study. One factor may be the absence of any association between S1 and responses: when seeing $\mathrm{X}$ or $\mathrm{U}$ in the combination task, participants did not know whether left or right key-presses would be required. If this factor is critical, then making S2 predictable based on S1 may allow for creating such associations and for activating the appropriate response already by perceiving $\mathrm{S} 1$, thereby for undoing the reduction of the oddball effect. This account will be tested in Experiment 1. Another factor may be complexity of S-R mappings. This complexity may result from the overlap of the same two responses with four different S1-S2 combinations (4:2 mapping): Perhaps, response mapping for the infrequent $U$ (e.g., left with yellow, right with blue) cannot be held in readiness because the responses "left" and "right" are continuously required for the frequent X. Moreover, this overlap may lead to conflicts between mapping of responses to S2 after rare S1 with such mappings after frequent S1. E.g., blue means left and yellow right for the frequent $\mathrm{X}$, but blue means right and yellow left for the infrequent $U$. This account in terms of complexity will be tested in Experiment 2: By gradually decreasing the S-R overlap between frequent and rare S1 and the interdependency of S1 and S2 in defining the responses, the oddball effect is expected to become gradually restored. Alternatively, the critical factor may not be difficulty of response selection but rather the increased relevance of S2 for determining the responses. This account will be tested in Experiment 3 by comparing the oddball and combination tasks to go/no-go tasks where S2 color will indicate whether the selected response should or should not be executed: With S2 being more relevant in the go/no-go tasks than in the oddball task and less relevant than in the combination task, the oddball effect on P3b is expected to be smaller in the go/no-go task than in the oddball task and larger than in the combination task.

A large negative potential appeared in the ERPs evoked by infrequent stimuli in Verleger, Baur, et al.'s (2014) combination task. So it could be suspected that P3b proper had remained unchanged and was only apparently reduced by overlap with this negative potential. But this negativity could be spatially and temporally dissociated from $\mathrm{P} 3 \mathrm{~b}$, because of its fronto-central focus, which was distinct from P3b's parietal focus, and by its being time-locked to onset of the S2 color frames following the infrequent S1, rather than to $\mathrm{S} 1$ onset. P3b remained reduced in that study even when, with lengthened intervals between S1 and S2, the increased negativity occurred much later than S1-evoked P3b. So the reduction of P3b was genuine. The negative potential was interpreted as a correlate of difficult response selection (cf. Hanslmayr et al., 2008; Johnson, Henkell, Simon, \& Zhou, 2008; Lang, Obrig, Lindinger, Cheyne, \& Deecke, 1990) and is expected to occur in the present study as well, whenever responses are difficult to select. stimuli

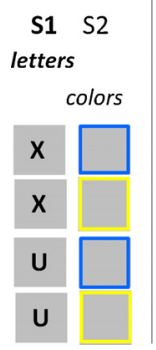

S2 irrelevant oddball (S1 80/20, S2 80/20)

left $\quad 64 \%$

left $16 \%$

right $16 \%$

right $4 \%$ tasks

\begin{tabular}{|c|c|c|c|}
\hline \multicolumn{4}{|c|}{$\mathrm{S} 2$ relevant } \\
\hline \multicolumn{2}{|c|}{$\begin{array}{c}\text { combination 50\% } \\
\text { (S1 80/20, S2 50/50) }\end{array}$} & \multicolumn{2}{|c|}{$\begin{array}{c}\text { combination } 80 \% \\
\text { (S1 80/20, S2 80/20) }\end{array}$} \\
\hline left & $40 \%$ & left & $64 \%$ \\
\hline right & $40 \%$ & right & $16 \%$ \\
\hline right & $10 \%$ & right & $16 \%$ \\
\hline left & $10 \%$ & left & $4 \%$ \\
\hline
\end{tabular}

Fig. 1. Outline of tasks in Experiment 1. Entered are percentages of occurrence of each stimulus in each task. Each task was performed twice, once with frequent Xs and once with frequent Us (in the first and second half of the experiment). Only the version with frequent Xs is depicted, for simplicity. See text for further description of the tasks.

\section{Experiment 1}

\subsection{Introduction}

In Experiment 1, we aimed at replicating the reduction of the oddball effect on S1-evoked P3b in the combination task (Verleger, Baur, et al., 2014) and at studying whether the reason for this reduction is the absence of associations between $\mathrm{S} 1$ and responses. The combination task was presented in two versions that differed from each other by the absence versus presence of associations between S1 and S2, thereby between S1 and responses. Making responses predictable might activate the S-R links with S1 already, which might restore the oddball effect on P3b. The stimuli consisted of the letters $\mathrm{X}$ and $\mathrm{U}(\mathrm{S} 1)$ and following blue or yellow color frames (S2), separated by onset asynchronies of $500 \mathrm{~ms}$. One of the two letters was frequently presented, the other rarely (80/20\%). Fig. 1 displays the stimuli and the assignments to left or right key-press responses in the three tasks.

One task was a simple oddball task with delayed responses. The letter $\mathrm{X}$ required a left response, $\mathrm{U}$ a right response, and S2 served as go-signal, independently of its color. The second task was the combination task used by Verleger, Baur, et al. (2014), except that stimulus onset asynchronies (SOAs) between S1 and S2 had been $0 \mathrm{~ms}, 100 \mathrm{~ms}, 400 \mathrm{~ms}$, or $800 \mathrm{~ms}$ in that study, while $500 \mathrm{~ms}$ SOA was used here. This is a "combination task" because information from S1 and S2 has to be combined to determine the response. Here, it will be termed "combination 50\%" to denote that blue and yellow S2s, and thereby left and right responses, were equally probable. The third task was "combination $80 \%$ " because, while response assignments were identical to combination $50 \%$, blue and yellow S2 were presented with probabilities of $4 / 1$, rather than $1 / 1$, such that a given letter as $S 1$ predicted the response with $80 \%$ probability (cf. Fig. 1). Thereby, combination $80 \%$ might be considered midway between oddball, where a given $\mathrm{S} 1$ predicted the response with $100 \%$, and combination $50 \%$, where a given S1 predicted the response with $50 \%$ only. Therefore, if reduction of the oddball effect on P3b in the combination task (Verleger, Baur, et al., 2014) was due to different predictive validities of S1 on responses, then probabilities of $80 \%$ might still produce a smaller oddball effect than the $100 \%$ S1-response probabilities in the oddball task but a larger one than with the $50 \%$ chance probabilities in combination $50 \%$.

We also expected to replicate the large S2-evoked negativity that accompanied the difficult response selections following infrequent S1 in Verleger, Baur, et al.'s (2014) combination task. Since response selection will be easier in combination $80 \%$ when $\mathrm{S} 1$ allowed for predicting the probable response, this S2-evoked negativity may be reduced in this task. 


\subsubsection{Participants}

Twelve young adults participated, eleven of which were university students They were 7 women and 5 men, aged $19-27$ years ( mean $=23, S D=2.2$ ). Informed written consent was obtained and $15 €$ were paid. Participants reported normal or corrected-to-normal vision, no history of neurological disorders, and being righthanded, as quantified by Edinburgh Handedness Inventory scores (Oldfield, 1971) of 70-100 (mean 92.5, SD 9.65).

\subsubsection{Stimuli and procedure}

Participants were seated in a comfortable armchair in a darkened room, with about $1.2 \mathrm{~m}$ viewing distance from the computer screen. A computer keyboard was put on their lap. Response keys were left ctrl and right ctrl, to be used with the index fingers. Controlled by a Presentation 14.5 program (www.neurobs.com) this computer presented the stimuli, recorded responses, and sent stimulus and response codes to the computer that recorded EEG.

Each trial started with a small black fixation cross, presented for $900 \mathrm{~ms}$ at the center of a light gray $17^{\prime \prime}$ screen. Then, one of the two black letters X and U (Helvetica, 35 points) appeared as $\mathrm{S} 1$, followed after an onset asynchrony of $500 \mathrm{~ms}$ by a blue or yellow rectangle as $\mathrm{S} 2$, surrounding the letter position $(2.3 \mathrm{~cm} \times 2.5 \mathrm{~cm}$ width $\times$ height, line width 3 pixels). S1 and S2 were presented for $200 \mathrm{~ms}$ each. $X$ and U varied across trials in random order with probabilities of $80 / 20 \%$ with a restriction against immediate repetition of rare letters. Blue and yellow S2s also varied across trials in random order, but with probabilities differing between tasks: 50/50\% in the combination $50 \%$ task, and $80 / 20 \%$ both in the combination $80 \%$ task and in the oddball task. (In this latter task, this variation was irrelevant.) Pressing the correct key after S2 onset terminated the trial, and the next S1 appeared $900 \mathrm{~ms}$ after this key-press. There were 250 trials within each block. Thus, with average RTs of, e.g., $400 \mathrm{~ms}$ and without any errors, a block would last for 250 trials $\times(900+500+400)$ $\mathrm{ms}=450 \mathrm{~s} \approx 71 / 2 \mathrm{~min}$. An error message appeared for $4 \mathrm{~s}$ in red $30 \mathrm{pt}$. font ("pressed too early", in German) when keys were pressed before S2 onset, which was important in the oddball task because responses were fully defined by S1 already. Blocks were preceded by instruction screens, informing about the S-R assignment in the following block

There are six possible orders for presenting the three tasks (oddball, combination $50 \%$, combination $80 \%$ ). Each order was used for two participants. Each task was presented in two versions, once with frequent $X$ and rare $U$ (as depicted in Fig. 1), and once with frequent $U$ and rare $X$. This way, unequal left-right response probabilities were balanced in the oddball and combination $80 \%$ tasks. The frequent- $X$ versions were presented as first three blocks to half the participants and as last three blocks to the other half.

\subsubsection{EEG recording and processing}

EEG was recorded with $\mathrm{Ag} / \mathrm{AgCl}$ electrodes (Easycap, www.easycap.de) from 60 scalp sites, including 8 midline positions from AFz to $\mathrm{Oz}$ and 26 pairs of symmetric left and right sites. Additional electrodes were placed at the nose-tip for off-line reference and at Fpz as connection to ground. On-line reference was Fz. For artifact control, EOG was recorded, vertically (VEOG) from above vs. below the right eye and horizontally (hEOG) from positions next to the left and right tails of the eyes. Data were amplified from DC to $250 \mathrm{~Hz}$ by a BrainAmp MR plus and stored at $500 \mathrm{~Hz}$ per channel. Off-line processing was done with Brain-Vision Analyzer software (version 2.03). Data were re-referenced to the nose-tip, low-pass filtered at $25 \mathrm{~Hz}$, and segmented from $100 \mathrm{~ms}$ before S1 onset to $1500 \mathrm{~ms}$ afterwards. To edit for artifacts, trials first were rejected as gross artifacts when consecutive data points differed by more than $50 \mu \mathrm{V}$ or when minimum and maximum of voltages in any EEG channel differed by more than $250 \mu \mathrm{V}$ (except EOG and AF3, AFz, AF4, lest trials would be rejected for blinks). Then, ocular artifacts were corrected by linear regression, using the method implemented in the Brain Analyzer software. Finally, data were referred to the mean amplitude of the first $100 \mathrm{~ms}$ as baseline in each channel, and trials were rejected when voltages exceeded $\pm 150 \mu \mathrm{V}$ in any EEG channel. The remaining trials were included when the first key-press responses were correct and made after S2 onset.

\subsubsection{Data analysis}

Response times (RTs) were analyzed for correct responses between $50 \mathrm{~ms}$ and $1000 \mathrm{~ms}$ after S2 onset. Left and right responses (i.e., blocks with frequent $\mathrm{X}$ and frequent $U$ ) were pooled and RTs were averaged across trials in each of the three tasks separately for frequent and rare S1s and for blue and yellow S2s (=frequent and rare S2s in oddball and combination $80 \%$ ). In the same way, percentages of wrong responses were determined (including too late responses, i.e., >1000 ms).

For analysis of S1-evoked P3b, trials were averaged separately for rare and frequent $\mathrm{S} 1$ in each task in each participant, pooling across blocks with frequent $\mathrm{X}$ and frequent $U$ and across trials with blue and yellow S2 (because S1-evoked P3b could not be affected by S2). The mean number of included trials for infrequent S1, separately for each of the three tasks, was 75 , with a minimum of 49 . Then, differences were formed between averages from rare and frequent S1. P3 was measured in these difference waveforms. Lacking a clear peak in the combination tasks, P3 amplitudes were measured in all tasks as mean amplitudes 300-600 ms after S1 onset.
For analyzing the central negativity in rare-frequent difference waveforms, data were additionally split by S2 color because response selection, reflected by this negativity, was expected to be more demanding in the combination $80 \%$ task with infrequent than frequent $S 2$. The mean number of included trials for the most infrequent category (4\% probability; infrequent S1 followed by infrequent S2) was 17 for oddball, 14 for combination $80 \%$, with a minimum of 12 and 8 , and 33 for combination $50 \%$, minimum 22 . This negativity was quantified by measuring mean amplitudes $250-450 \mathrm{~ms}$ after S2 onset (750-950 ms after S1 onset).

All measurements were taken in data of the seven midline channels $\mathrm{Fz}, \mathrm{FCz}, \mathrm{Cz}$, $\mathrm{CPz}, \mathrm{Pz}, \mathrm{POz}, \mathrm{Oz}$.

Analyses of variance (ANOVAs) were used for statistical analyses. Pair-wise comparisons were conducted, on the one hand, between the oddball and combination $50 \%$ tasks in order to test whether Verleger, Baur, et al.'s (2014) results could be replicated, and, on the other hand, between the combination $50 \%$ and $80 \%$ tasks in order to test whether the reduction of the oddball effect could be undone by the $80 \%$ association from S1 to responses. Repeated measurement factors for analysis of RTs and of error rates were S1 Frequency (frequent vs. rare), Task (oddball vs. combination $50 \%$ in one analysis, combination $50 \%$ vs. combination $80 \%$ in the other), and S2 Color (blue vs. yellow; color variation was irrelevant in the oddball task and relevant in the two combination tasks, where blue and yellow were presented with 80/20 probabilities in the combination $80 \%$ task, and with $50 / 50$ probabilities in the combination $50 \%$ task). For analysis of P3, factors were Recording Site ( $\mathrm{Fz}, \mathrm{FCz}, \mathrm{Cz}, \mathrm{CPz}, \mathrm{Pz}$, $\mathrm{POz}, \mathrm{Oz}$ ) and Task (as above). For analysis of central negativity, factors were Recording Site, Task, and S2 Color (all defined as above). To interpret interactions, ANOVAs were conducted separately for the levels of each of the interacting factors. Degrees of freedom of the Recording Site factor (being a repeated-measurement factor with more than two levels) were corrected with the Greenhouse-Geisser method.

\subsection{Results}

\subsubsection{S1-evoked P3}

Grand mean waveforms are displayed in Fig. 2. The oddball effect was obviously larger in oddball than in combination 50\% (Task: $\left.F_{1,11}=25.7, p<.001\right)$. Crucially, this reduced effect did not differ between the two combination tasks (Task: $F_{1,11}=0.0$, n.s.).

The oddball effect on P3 was largest at Pz (cf. the maps of P3's topographical distribution at the bottom of Fig. 2; effects of Recording Site: $F_{6,66}=16.3, p<.001$ in oddball vs. combination $50 \%$, and $F_{6,66}=8.1, p=.001$ in combination $50 \%$ vs. combination $80 \%$ ). Topographies of the oddball effect differed between the oddball and combination 50\% tasks (Task $\times$ Recording Site: $F_{6,66}=8.7, p=.002$ ) but this difference disappeared after vector scaling (i.e., normalizing the amplitudes in order to compare the topographic profiles independently of amplitude differences), $F_{6,66}=0.4$, n.s., which may speak against a true change (McCarthy \& Wood, 1985, though see Urbach \& Kutas, 2006). Topographies did not differ between the two combination tasks (Task $\times$ Recording Site: $F_{6,66}=0.8$, n.s.).

\subsubsection{S2-evoked negativity}

2.3.2.1. Oddball vs. combination $50 \%$. Negativity was larger at anterior than posterior sites (Recording Site: $F_{6,66}=5.4, p=.008$ ) but was restricted to $\mathrm{Fz}$ and $\mathrm{FC} z$ in the oddball while extending from Fz backwards to $\mathrm{Pz}$ in combination 50\% (Task $\times$ Recording Site, $F_{6,66}=3.8$, $p=.02$ ) such that negativity was larger in combination $50 \%$ than in oddball at $\mathrm{Cz}, \mathrm{CPz}$, and Pz. (Effect of Task in separate analyses at these recording sites: $F_{1,11} \geq 6.2, p \leq .03$ ). There was no effect of S2 Color $(F \leq 2.1, p \geq .13)$.

\subsubsection{Combination 50\% vs. combination $80 \%$. Negativity was} appreciably larger after the $20 \%$ probability (yellow) S2 in combination $80 \%$ than after the $80 \%$ probability (blue) S2, which did not differ from the $50 \%$ probability S2s in the combination 50\% task, resulting in a Task $\times$ S2 Color interaction, $F_{1,11}=9.6, p=.01$, and main effects of Task, $F_{1,11}=9.7, p=.01$, and of S2 Color, $F_{1,11}=9.2$, $p=.01$. Negativity extended with a flat topography from $\mathrm{Fz}$ to $\mathrm{Pz}$ after blue (frequent) S2s, and increased above all from $\mathrm{FCz}$ to $\mathrm{Pz}$ with yellow (rare) S2s. The effect was reflected in a main effect of Recording Site, $F_{6,66}=6.3, p=.002$, and an S2 Color $\times$ Recording Site interaction, $F_{6,66}=3.3, p=.03$, unmodified by Task. 


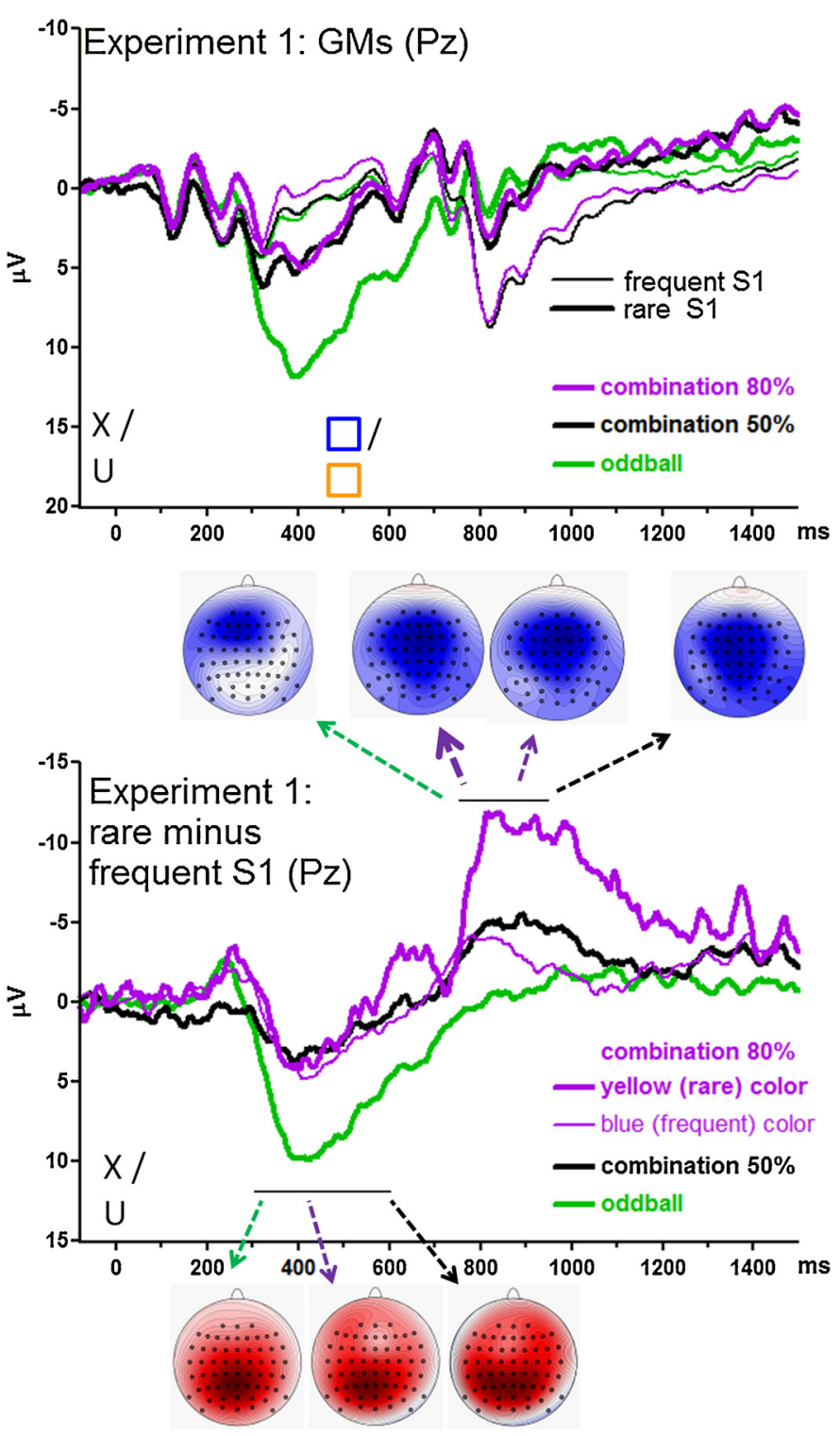

Fig. 2. Grand means of ERPs from Pz in Experiment 1. Unit of $x$-axis is ms. Letters were presented at time-point $0 \mathrm{~ms}$, and colored frames at $500 \mathrm{~ms}$. Unit of $y$-axis is $\mu \mathrm{V}$, with negative polarity plotted upwards and scales spanning $30 \mu \mathrm{V}$ equally in both panels. Green lines denote data from the Oddball 4/1 task, black lines from Combination $1 / 1$ and purple lines from Combination $2 / 1$. In the upper panel, data are displayed separately for frequent S1 (thin lines) and rare S1 (bold lines). Difference waveforms rare-frequent S1 are displayed in the lower panel, with data from the Combination 2/1 task split by S2 color, with the frequent S2 color in bold and the rare S2 color as thin line. Topographies of the difference peaks are displayed for mean amplitudes 300-600 ms after S1 (below the waveforms) and 250-450 ms after S2 (above the waveforms). Colors and styles of arrows denote the conditions. Positive polarity is red, negative polarity is blue, scaling is from minimum to maximum, differing between maps. (For interpretation of the references to color in this figure legend, the reader is referred to the web version of the article.)

\subsubsection{Response times and error rates}

Mean RTs and error rates are displayed in the left panels of Fig. 3.

2.3.3.1. Oddball vs. combination $50 \%$. Responses were much slower and more errors were committed in combination $50 \%$ than in the oddball, $F_{1,11}=84.4, p<.001$ for RT, $F_{1,11}=32.0, p<.001$ for error rate. It was only in combination $50 \%$ that responses were slower (by about $100 \mathrm{~ms}$ ) and more errors were committed (about $16 \%$ more) after rare than after frequent $S 1$, reflected by main effects of S1 Frequency, $F_{1,11}=57.2, p<.001$ for RT, $F_{1,11}=32.2, p<.001$ for errors, and by Task $\times$ Frequency interactions, $F_{1,11}=59.0, p<.001$ for RT, $F_{1,11}=24.8, p<.001$ for errors, and consequently by effects of Frequency being significant in the combination $50 \%$ task only, $F_{1,11}=89.0, p<.001$ for RT, $F_{1,11}=29.3, p<.001$ for errors, not in the oddball task, $F_{1,11} \leq 0.2$, n.s for RT and errors. Effects of S2 Color were not significant for errors, $F_{1,11} \leq 1.7, p \geq .22$, but RTs were, unexpectedly, slower in the combination task to yellow than to blue S2s (although either color had 50\% probability), reflected by a main effect of S2 Color, $F_{1,11}=8.9, p=.01$, a Task $\times$ S2 Color interaction, $F_{1,11}=6.9, p=.02$, and the effect of Color being significant in the combination $50 \%$ task, $F_{1,11}=8.7, p=.001$, not in the oddball task, $F_{1,11}=0.6$, n.s.

2.3.3.2. Combination 50\% vs. combination $80 \%$. As reflected by main effects of S1 Frequency, responses were slower and much more errors were committed after rare than after frequent $S 1$, $F_{1,11}=133.9, p<.001$ for RT, $F_{1,11}=50.7, p<.001$ for errors. The effects of $S 2$ Color, $F_{1,11}=34.4, p<.001$ for RT, $F_{1,11}=14.6, p=.003$ for errors, and of Task $\times$ S2 Color, $F_{1,11}=28.7, p<.001$ for RT, $F_{1,11}=11.2$, $p=.007$ for errors, reflected large effects of Color in the combination $80 \%$ task, $F_{1,11}=44.4, p<.001$ for RT, $F_{1,11}=23.7, p<.001$ for errors. These effects meant that, after blue $\mathrm{S} 2$, responses became faster and error rates smaller in combination $80 \%$ than in combination $50 \%$ ( $80 \%$ vs. $50 \%$ probability of blue $S 2$ ), $F_{1,11}=23.5, p=.001$ for RT and $F_{1,11}=5.6, p=.04$ for errors for the effect of Task in separate analysis on blue S2. There was no difference between tasks when S2 was yellow ( $20 \%$ vs. $50 \%$ probability in these two tasks), $F_{1,11} \leq 0.6$, n.s. for RT and errors. For RTs, the speeding of responses with blue S2 in combination $80 \%$ was larger after frequent than after infrequent S1 ( $67 \mathrm{~ms}$ vs. $38 \mathrm{~ms}$ ), as indicated by the triple interaction of Task $\times$ S2 Color $\times$ S1 Frequency, $F_{1,11}=18.3, p=.001$, resolving to a significant interaction of Task $\times S 1$ Frequency with blue $S 2, F_{1,11}=5.5, p=.04$, and not with yellow $S 2, F_{1,11}=1.7$, n.s. This triple interaction was not significant for errors, $F_{1,11}=1.5$, n.s.

\subsection{Discussion}

Increasing the probability of one S2 color, in the combination $80 \%$ task, speeded up RTs and decreased error rates compared to the combination 50\% task. Since responses to this color were defined by the specific S1-S2 (letter-color) combination rather than by the $\mathrm{S} 2$ color, this result shows that associations between letter S1 stimuli and responses were successfully established in the combination $80 \%$ task. Nevertheless, the S1-evoked oddball effect on P3 amplitudes was as reduced in this task as in the original combination (50\%) task. Therefore, no evidence was found for the assumption that the reduction of the oddball effect in the combination task is due to the absence of associations between S1 stimuli and responses.

It came unexpected that there was a similar, albeit much smaller effect of S2 color on RTs in the combination 50\% task, with responses being faster to blue than to yellow frames. This might have been due to a primacy effect in instructions, because participants were always given the instruction on blue S2 first (e.g., " $\mathrm{X}$ and blue means left, $X$ and yellow means right"). Alternatively, it might be due to carryover when combination $50 \%$ was preceded by combination $80 \%$. To test for this latter possibility, additional analyses distinguished between those participants who had combination $80 \%$ before combination $50 \%$, and vice versa, and between the versions of the combination $50 \%$ task presented within the last three blocks (such that at least one combination 50\% task preceded) vs. the first three blocks. But none of these order factors interacted with S2 Color. Thus, the primacy effect in instructions might have been decisive, cf. Kotchoubey (2014) for a related finding and more thorough discussion. 


\section{Experiment 1}
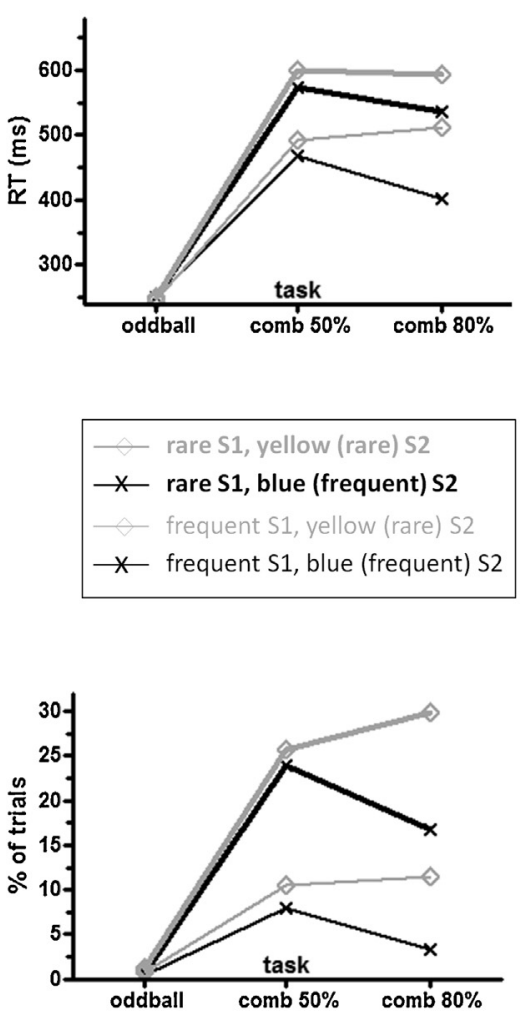

Experiment 2

\section{response times}
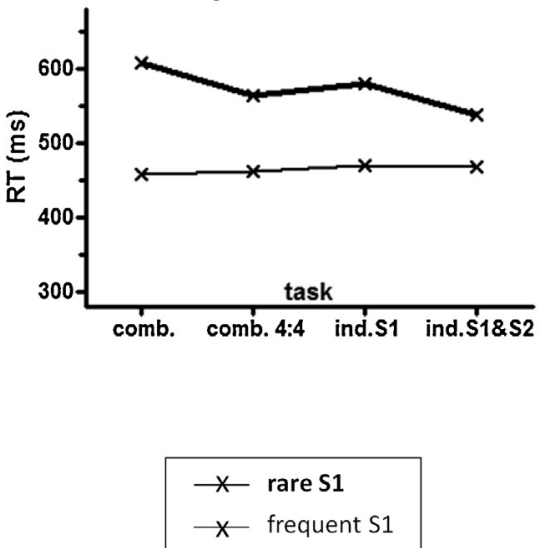

error rates

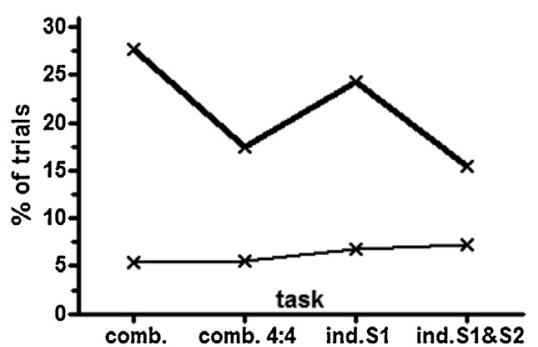

Experiment 3
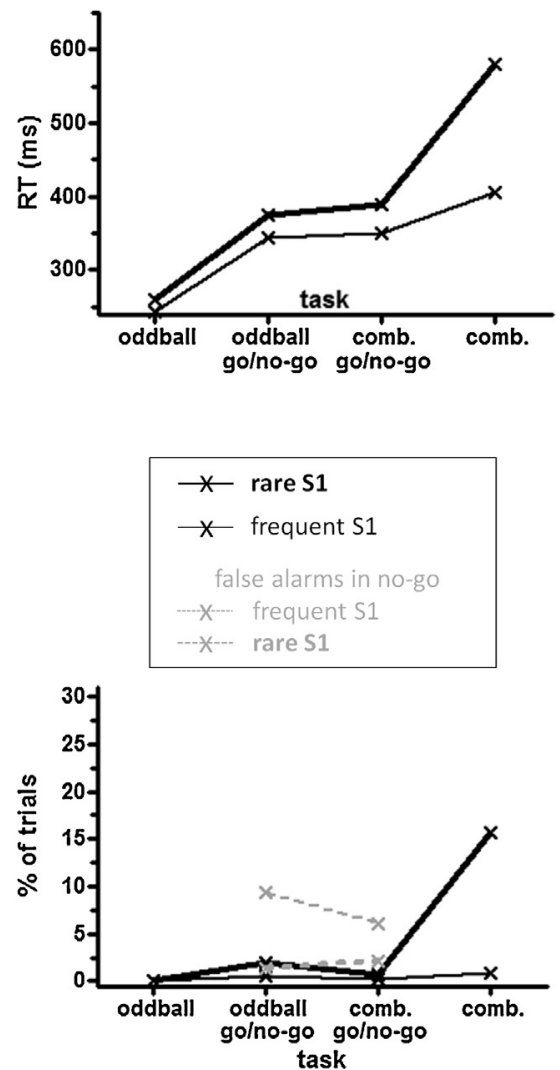

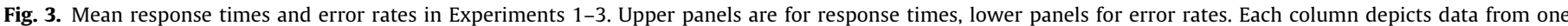

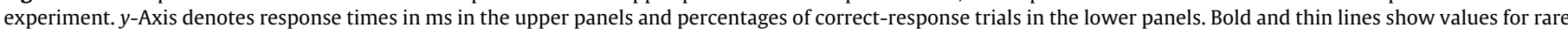

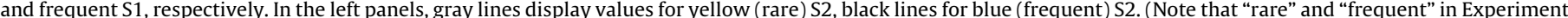

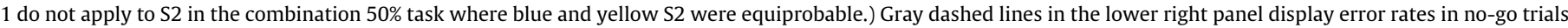
(i.e., false alarms), with bold and thin lines used for rare and frequent $\mathrm{S} 1$, respectively.

\section{Experiment 2}

\subsection{Introduction}

Experiment 2 investigated whether the reduction of the oddballP3b in the combination task of Verleger, Baur, et al. (2014) was due to complexity of S-R mappings. Three tasks were implemented that reduced this complexity compared to the original combination task. By stepwise decreasing complexity across tasks the oddball effect is expected to become gradually restored. Unlike in Experiment 1, stimulus probabilities were identical across the four tasks. Fig. 4 displays the stimuli and their response assignments in its upper part, and provides a survey on the factors presumed to contribute to reduction of the oddball effect in its lower part, as detailed in the following.

The combination task used by Verleger, Baur, et al. (2014) and in Experiment 1 channels the four possible combinations of $\mathrm{S} 1 \times \mathrm{S} 2$ to two responses (therefore will here be called "combination 4:2"): Left-hand responses are required both by blue S2 after X and by yellow $S 2$ after $U$, and right-hand responses are required both by yellow $S 2$ after $X$ and by blue $S 2$ after $U$. If this $4: 2$ mapping is critical for reducing the oddball effect then the oddball effect should be smaller with this task than with the three other tasks, in which four rather than two fingers were used for responding. To be better comparable to the other tasks where middle fingers and thumbs were used for responding, this "combination 4:2" task was run twice, once with thumbs and once with middle fingers as responding agents.

Among the three other tasks, most similar to the original task was the "combination 4:4" task: The critical difference was that overlap of responses was removed by assigning different responses to each S1-S2 combination. Therefore, as just noted, if oddball-P3b was reduced in the original combination task because responses to rare S1 cannot be independently stored in working memory due to their overlapping with responses assigned to frequent $\mathrm{S} 1$, then this reduction should not occur any more in the combination 4:4 task.

In the "independent S1" task, letters provided independent response-relevant information. E.g., an $\mathrm{X}$ may mean that the middle finger will be used, and a U may mean "thumb". Which hand to use was specified by the following S2, still to be combined with S1 information and still having opposite meanings for rare vs. frequent S1 (e.g., blue S2 means "left" after X and “right" after U). Therefore, if oddball-P3b was reduced in the combination task because the infrequent $\mathrm{S} 1$ did not provide any independent information, then this reduction should not occur any more in the independent-S1 task.

So far, in all tasks, S2 information had opposite meanings with the two S1s. This feature was abolished in the "independent S1\&S2" task. Here, letter and color provided independent information, such that, e.g., after having specified the middle finger (like in the previous task), the $\mathrm{X}$ information did not need to be combined with the S2 information. (Thus, this task is a pre-cueing task as devised 


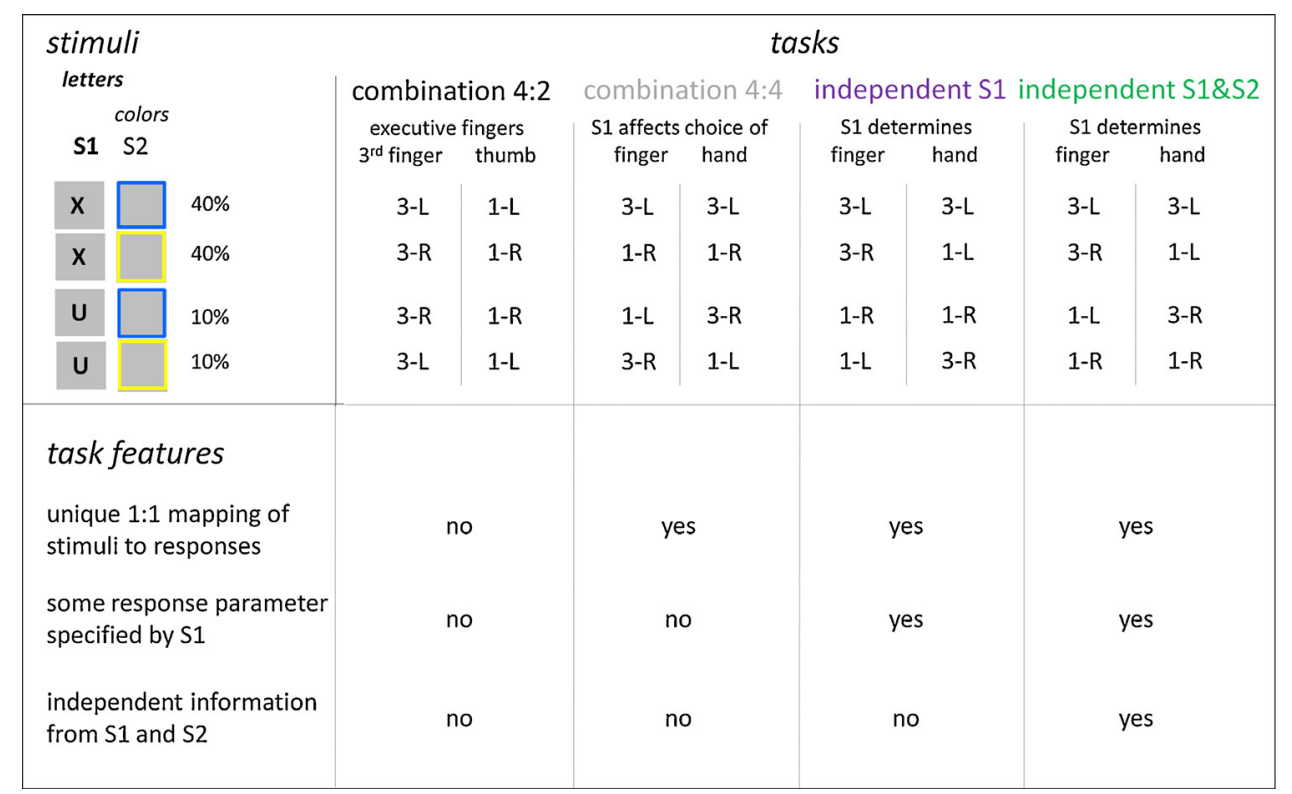

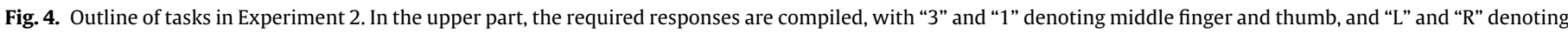

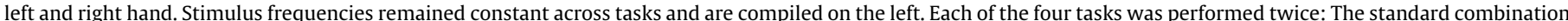

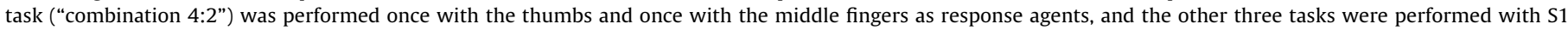

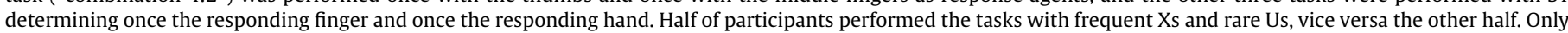

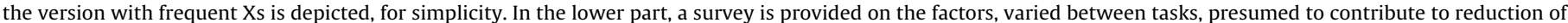
the oddball effect. See text for further description.

by Rosenbaum, 1980.) Therefore, if oddball-P3b was reduced in the combination task because information from infrequent S1 still had to be stored for further combination with S2 information, then this reduction should not occur any more in the independent S1\&S2 task.

\subsection{Material and methods}

Differences from Experiment 1 will be described only.

\subsubsection{Participants}

Fourteen university students participated, but two of them had to be excluded due to malfunctions of experimental control. The remaining 12 participants were 7 women and 5 men, aged $19-26$ years (mean $=22.6, S D=2.0$ ). Informed written consent was obtained and $5 €$ base remuneration was paid plus $1 \mathrm{ct}$. for each correct response, adding together to amounts around $20 €$. Participants reported normal or corrected-to-normal vision, no history of neurological disorders, and being righthanded, as quantified by Edinburgh Handedness Inventory scores of 80-100 (mean 88, SD 9.4).

\subsubsection{Stimuli and procedure}

Response keys were Tab for the left middle finger, Left Ctrl for the left thumb, Enter for the right middle finger, and Right Ctrl for the right thumb. S2 probabilities were identical in all tasks, 50/50. Because all tasks were difficult, blocks were preceded by instruction screens, denoting the stimulus-response assignment in the following block, followed by ten practice trials. Correct responses were rewarded by $1 \mathrm{ct}$. each. Feedback screens after every 50 trials informed about the number of correct responses and the amount of money earned.

There were four tasks, as described above: combination 4:2 (as in Experiment 1 and in Verleger, Baur, et al., 2014), combination 4:4, independent S1, and independent S1\&S2. Each task was presented in two versions, as detailed in Fig. 4.

The two versions of each task were principally presented in consecutive blocks, except that the combination 4:2 task was always presented in the middle, in the 4 th and 5 th blocks, thereby splitting the two versions of the middle one of the three other tasks to become blocks \#3 and \#6. The order of these three other tasks was fully balanced: Each of the 6 possible permutations was used in two participants, with one participant having each task's "finger" version followed by its "hand" version, vice versa in the other participant. Crossed with this splitting, half the participants had $\mathrm{X}$ as frequent and $\mathrm{U}$ as rare stimuli (as depicted in Fig. 4) and had the middle-finger version of the combination 4:2 task before the thumb version, and the other half had $U$ as frequent and $X$ as rare stimuli and the thumb version of the combination $4: 2$ task before the middle-finger version.

\subsubsection{EEG recording and processing}

Methods were identical to Experiment 1.

\subsubsection{Data analysis}

RTs and error rates of the two versions of each task were compared to each other in preliminary analyses, and no differences were found. For the main analyses, data were pooled across the two versions, and pooled across trials with blue and yellow S2. Likewise, for ERP analysis, trials were averaged separately for rare and frequent $\mathrm{S} 1$ in each of the four tasks in each participant, pooling across trials with blue and yellow S2 and across the two versions of each task. The mean number of included trials, separately for each task, was 71 for infrequent S1, with a minimum of 34. P3 amplitudes were measured as mean amplitudes 350-600 ms after S1 onset.

Repeated measurement factors in ANOVAs were S1 Frequency (frequent vs. rare) and Task (combination 4:2, combination 4:4, independent $S 1$, and independent S1\&S2) for analysis of RTs and of error rates, and Recording Site (Fz, FCz, Cz, $\mathrm{CPz}, \mathrm{Pz}, \mathrm{POz}, \mathrm{Oz}$ ) and Task (as above) for analysis of $\mathrm{P} 3$ and Central Negativity in the difference waveforms of rare minus frequent $\mathrm{S} 1$.

\subsection{Results}

\subsubsection{S1-evoked P3}

Grand mean waveforms are displayed in Fig. 5. The oddball effect on P3 was largest at Pz and POz (Recording Site: $F_{6,66}=9.8, p<.001$ ). There was no difference between tasks, main effect $F_{3,33}=1.1$, n.s., Task $\times$ Recording Site, $F_{18,198}=1.2$, n.s.

\subsubsection{S2-evoked negativity}

The oddball effect on response selection, reflected in S2-evoked negativity, was largest at $\mathrm{FCz}$ and $\mathrm{Fz}$ (Recording Site: $F_{6,66}=5.7$ $p=.02$, Task $\times$ Recording Site, $F_{18,198}=1.1$, n.s.). Of interest, negativity differed between tasks (main effect of Task: $F_{3,33}=3.7, p=.04$ ). Pair-wise ANOVAs on each task pair showed that negativity was larger in combination 4:2 than in the two "independent" tasks, $F_{1,11} \geq 8.8, p \leq .01$, and weakly tended to be so compared to combination $4: 4, F_{1,11}=2.5, p=.14$. The three other tasks did not differ from each other, $F_{1,11} \leq 1.8, p \geq .21$.

\subsubsection{Response times and error rates}

Mean RTs and error rates are displayed in the middle column of Fig. 3. Responses were much slower and more errors were 


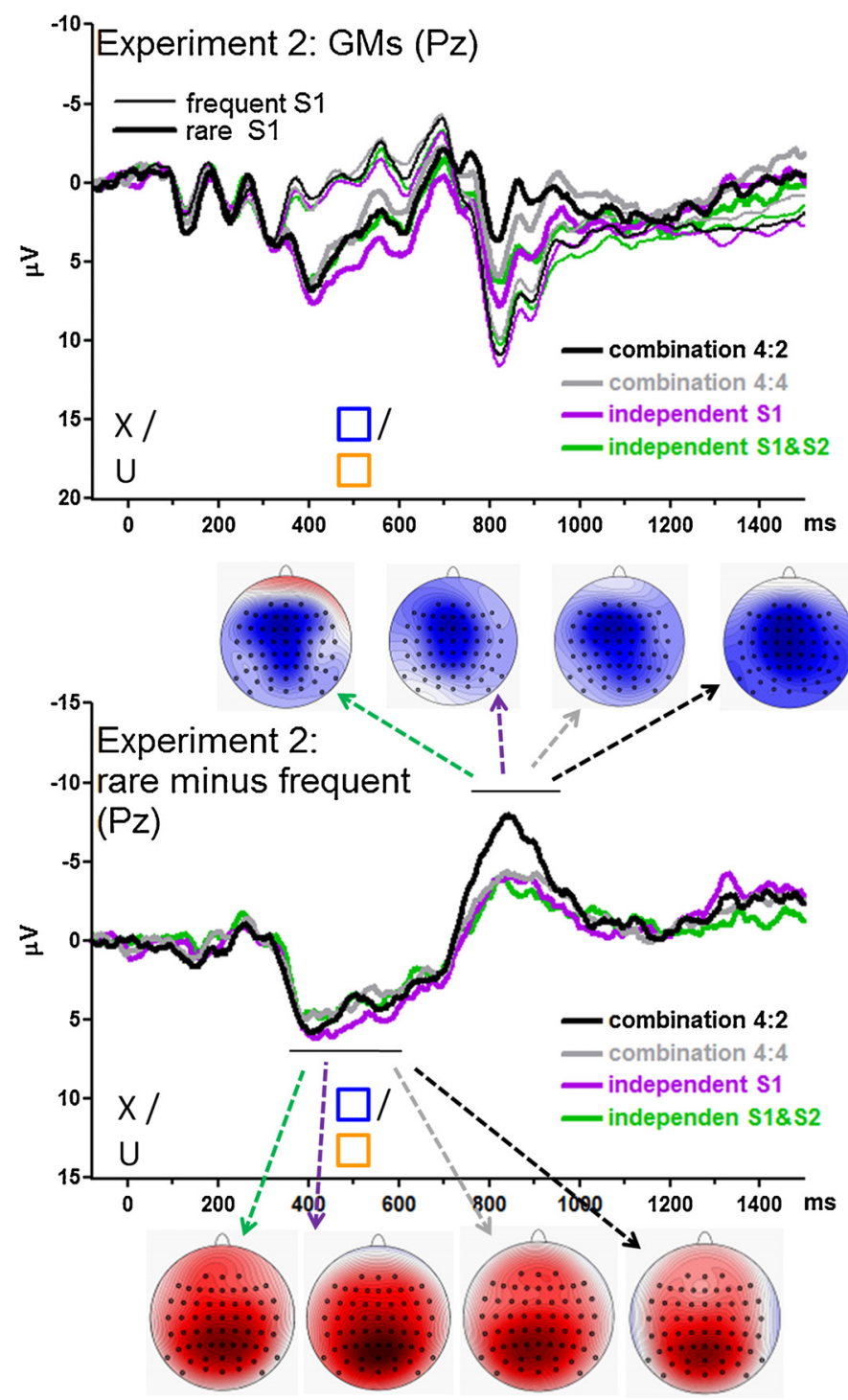

Fig. 5. Grand means of ERPs from Pz in Experiment 2. Unit of $x$-axis is ms. Letters were presented at time-point $0 \mathrm{~ms}$, and colored frames at $500 \mathrm{~ms}$. Unit of $y$-axis is $\mu \mathrm{V}$, with negative polarity plotted upwards and scales spanning $30 \mu \mathrm{V}$ equally in both panels. Black lines denote data from the Combination ("4:2") task, gray lines from Combination 4:4, purple lines from the "Independent" task, and green lines from the "Orthogonal" task. In the upper panel, data are displayed separately for frequent S1 (thin lines) and rare S1 (bold lines). Difference waveforms rare-frequent S1 are displayed in the lower panel. Topographies of the difference peaks are displayed for mean amplitudes 350-600 ms after S1 (below the waveforms) and 250-450 ms after S2 (above the waveforms). Colors of arrows denote the conditions. Positive polarity is red, negative polarity is blue, scaling is from minimum to maximum, differing between maps. (For interpretation of the references to color in this figure legend, the reader is referred to the web version of the article.)

committed after rare than after frequent $\mathrm{S} 1\left(F_{1,11}=220.9, p<.001\right.$ for RTs; $F_{1,11}=40.9, p<.001$ for error rates). This effect differed between tasks, both for RTs and for error rates, Frequency $\times$ Task, $F_{3,33}=7.0, p=.006$ for RTs, $F_{3,33}=6.0, p=.01$ for error rates. To localize these effects, rare- minus frequent-S1 differences were formed for each task, and these oddball effects on RTs and error rates were compared by $t$-tests between each pair of tasks. For RTs, oddball effects were smaller in the independent S1\&S2 task ( $70 \mathrm{~ms})$ than in all other tasks, $t_{11} \geq 2.9, p \leq .015$ and larger in the combination $4: 2$ task (151 ms) than in the independent S1\&S2 task and than in the combination $4: 4$ task, $t_{11} \geq 2.3, p \leq .04$, but not significantly so when compared to the independent $\mathrm{S} 1$ task, $t_{11}=1.7, p=.12$. Combination $4: 4(101 \mathrm{~ms})$ and independent S1 (112 ms) lay in the middle and

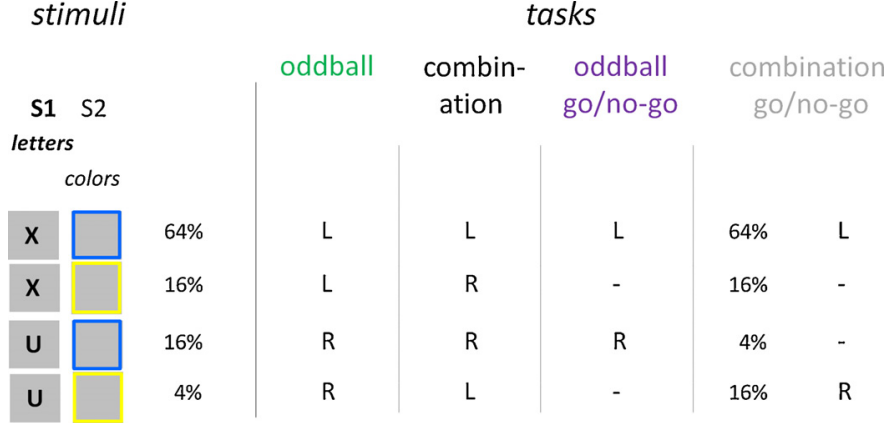

Fig. 6. Outline of tasks in Experiment 3. Entered are the required responses, with "L", "R", “-" denoting left hand, right hand, and no response, respectively. Stimulus frequencies, compiled at the left, remained constant across tasks except for reversal of S2 frequencies in the "combination go/no-go task", compiled at this task on the right. Half of participants performed the tasks with frequent Xs and rare Us, vice versa the other half. Only the version with frequent Xs is depicted, for simplicity. See text for further description.

did not differ from each other, $\mathrm{t}_{11}=0.8$, n.s. For error rates, oddball effects were relatively small in the independent $\mathrm{S} 1 \& \mathrm{~S} 2 \mathrm{task}(8 \%)$ and in the combination $4: 4$ task (12\%) and relatively large in the combination 4:2 task (22\%) and in the independent S1 task (17\%): There was no difference within either of these two pairs, $t_{11} \leq 1.6, p \geq .14$, while each member of the one pair differed from each member of the other pair, $t_{11} \geq 2.4, p \leq .04$.

\subsection{Discussion}

These four tasks of Experiment 2 differed from each other in the ways of how responses were mapped to S1-S2 combinations. Consequently, these four tasks differed in how difficult it was to select the response when $\mathrm{S} 1$ was the rare event, as indicated by three parameters: response delays, increased error rates, and increased S2-evoked fronto-central negativity. According to all three parameters, the original combination $(4: 2)$ task was the most difficult one. Nevertheless, the oddball effect on S1-evoked P3 did not change between tasks. Therefore, it may be concluded that even the easiest task, which was the independent S1\&S2 task (according to the a priori rationale and to behavioral effects) fully contained the factor that is responsible for the reduction of the oddball effect with the combination task. Still it remains unclear what this factor might be.

\section{Experiment 3}

\subsection{Introduction}

The preceding variations from the combination task had not restored at least some part of the reduction of the oddball-P3b, in spite of increased response predictability (Experiment 1) or reduced complexity of S-R mappings (Experiment 2). Therefore, these factors, all related to task difficulty, probably do not cause the reduction of oddball P3b. A more general feature distinguishing between the oddball task and all other tasks used so far is that in the oddball task S2 did not play any role in specifying the responses. Therefore, relevance of S2 for response selection will be varied in Experiment 3 by comparing the oddball and combination tasks to go/no-go tasks. In go/no-go tasks, S2 does not modify the choice prompted by $S 1$ but determines whether the selected response will or will not be executed. With $S 2$ being more relevant in the go/no-go tasks than in the oddball task and less relevant than in the combination task, the oddball effect on S1-evoked P3b is expected to be smaller in the go/no-go task than in the oddball task and larger than in the combination task. 
Therefore, as depicted in Fig. 6, Experiment 3 compared go/nogo tasks to the oddball task and to the combination task. Two versions of go/no-go tasks were used. In one version, termed here "oddball go/no-go", S2's meaning did not depend on S1, i.e., blue S2 always meant "go", and yellow S2 always meant "no-go". In the other version, termed "combination go/no-go", S2's meaning depended on S1, similar to the combination task, i.e., after X, blue S2 meant "go", and yellow S2 "no-go", while after U, yellow S2 meant "go" and blue S2 "no-go". In order to keep participants engaged with the task, the proportion of "go" and "no-go" signaled by S2 was $4 / 1(80 / 20)$. The same proportion of S2 stimuli was used in the oddball and in the combination task which, thus, was identical to Combination 80\% from Experiment 1.

\subsection{Material and methods}

Differences from the previous experiments will be described only.

4.2.1. Participants

Twelve university students participated. One original participant did not care about waiting for S2 before pressing and was, therefore, replaced by another one. They were seven women and five men, aged 23-27 years (mean $=25, \mathrm{SD}=2.9$ ) for $15 €$ remuneration. Participants were right-handed, as quantified by Edinburgh Handedness Inventory scores of 50-100 (mean 91, SD 15.1).

\subsubsection{Stimuli and procedure}

Response keys were left and right ctrl, like in Experiment 1. There were twenty practice trials before each task. There were four tasks, as described above: oddball, combination, oddball go/no-go, combination go/no-go. Probabilities of X and $U$ as S1 were 80/20 in half the participants (as displayed in Fig. 6), and 20/80 in the other half, meaning that in all tasks left-hand responses were frequent in the former group and right-hand responses in the latter. Probabilities of blue and yellow S2 were 80/20, equally across participants, though not equally across tasks: Whereas blue was presented with $80 \%$ and yellow with $20 \%$ frequency in three tasks, this proportion had to be reversed after $\mathrm{U}$ as $\mathrm{S} 1$ in combination go/no-go, to maintain an 80/20 proportion of go and no-go trials. We considered this an acceptable irregularity because the focus of this study lies on S1-evoked P3b rather than on effects evoked by S2.

Half the participants had the two go/no-go tasks in the middle, half as first and last. Half the participants had the oddball task before the combination task, vice versa in the other half, and half the participants had the oddball go/no-go task before the combination go/no-go task, vice versa in the other half.

\subsubsection{EEG recording and processing}

Methods were identical to Experiment 1 except that correctly responded no-go trials, to be included in the averages, were defined as trials without key-pressing.

\subsubsection{Data analysis}

Because infrequent-S2 trials were no-go trials in the go/no-go tasks, RTs of correct responses and percentages of missing responses were analyzed for frequent-S2 trials only. Additionally, percentages of false alarms were analyzed from the no-go trials.

For ERP analysis, trials were averaged separately for rare and frequent S1 in each of the four tasks in each participant, pooling across trials with rare and frequent S2. The mean number of included trials, separately for each of the four tasks, was 43 for infrequent $\mathrm{S} 1$, with a minimum of 25.

ANOVA factors were S1 Frequency (frequent vs. rare), Task (four levels), and Recording Site (Fz, FCz, Cz, CPz, Pz, POz, Oz), like in Experiment 2. When the fourlevel Task factor had effects in omnibus analyses, the a priori hypotheses were tested by ANOVAs conducted on all pairs of tasks.

\subsection{Results}

\subsubsection{S1-evoked P3}

Grand mean waveforms are displayed in Fig. 7. Overall, the oddball effect on P3 was largest at Pz (Recording Site: $F_{6,66}=17.6$, $p<.001$ ) and differed between tasks, main effect of Task $F_{3,33}=10.1$, $p<.001$, Task $\times$ Recording Site $F_{18,198}=3.4, p=.02$. Pair-wise analysis of tasks showed that oddball effects were larger in the oddball task than in the combination task, as could be expected, $F_{1,11}=27.9$, $p=.001$. Of much interest, the oddball effects in the two go/no-go tasks lay in-between the oddball task and the combination task: Effects in the go/no-go tasks were smaller than in the oddball, both in oddball go/no-go, $F_{1,11}=14.1, p=.003$, and in combination go/nogo $F_{1,11}=11.7, p=.006$; and larger than in the combination task, both in oddball go/no-go, $F_{1,11}=4.6, p=.05$, and at $\mathrm{Pz}$ in combination

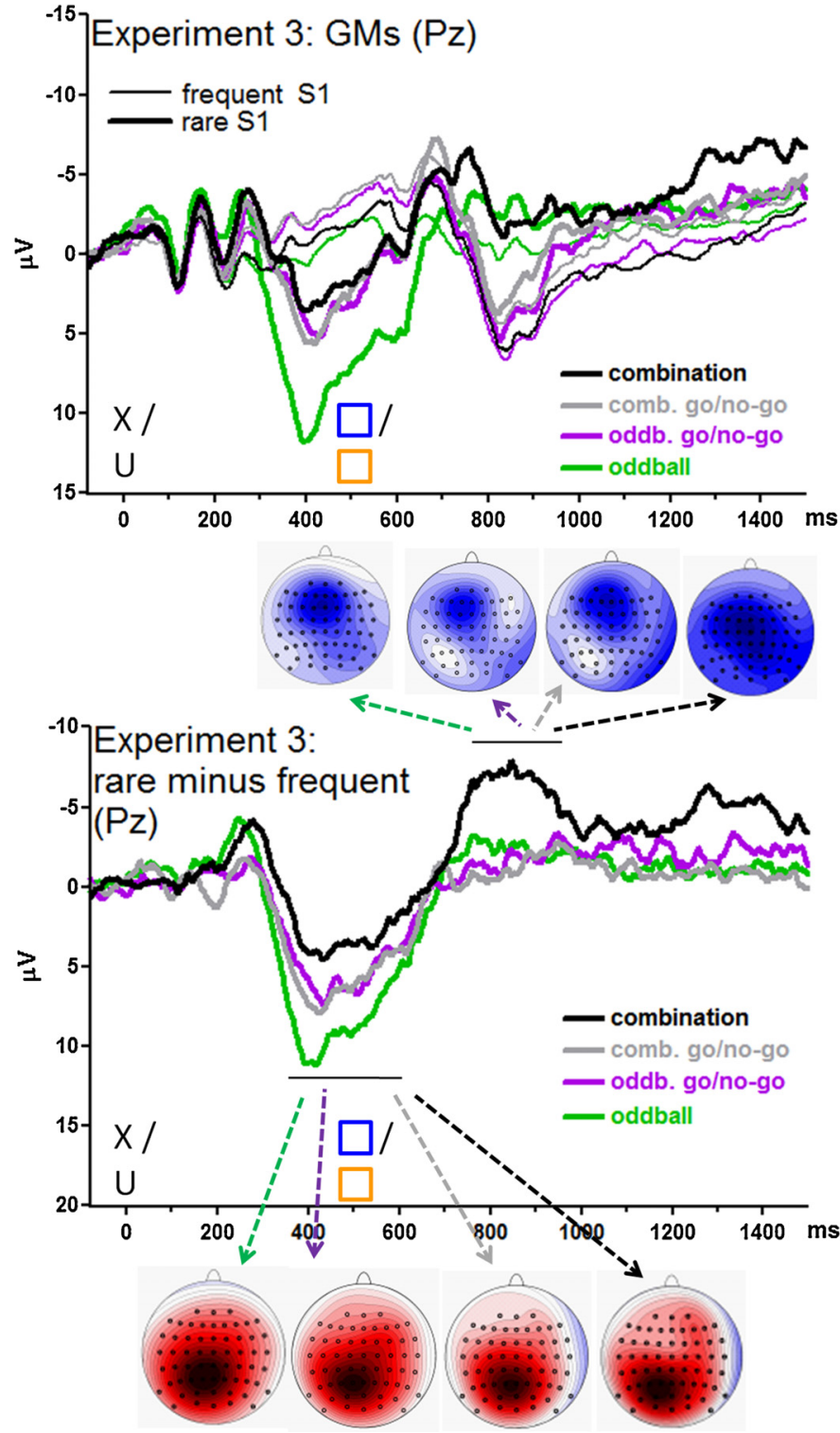

Fig. 7. Grand means of ERPs from Pz in Experiment 3. Unit of $x$-axis is ms. Letters were presented at time-point $0 \mathrm{~ms}$, and colored frames at $500 \mathrm{~ms}$. Unit of $y$-axis is $\mu \mathrm{V}$, with negative polarity plotted upwards and scales spanning $30 \mu \mathrm{V}$ equally in both panels. Black lines denote data from the Combination ("4:2") task, gray lines from Combination Go/No-Go, purple lines from Oddball Go/No-Go, and green lines from the Oddball task. In the upper panel, data are displayed separately for frequent S1 (thin lines) and rare S1 (bold lines). Difference waveforms rare-frequent S1 are displayed in the lower panel. Topographies of the difference peaks are displayed for mean amplitudes 350-600 ms after S1 (below the waveforms) and 250-450 ms after S2 (above the waveforms). Colors of arrows denote the conditions. Positive polarity is red, negative polarity is blue, scaling is from minimum to maximum, differing between maps. (For interpretation of the references to color in this figure legend, the reader is referred to the web version of the article.)

go/no-go $\left(F_{6,66}=5.0, p=.02\right.$, for the interaction of Task $\times$ Recording Site, which could be resolved to a Task effect at $\mathrm{Pz}, F_{1,11}=6.4$, $p=.03$ )

Oddball effects did not differ between the two go/no-go tasks, $F_{1,11}=0.2$, n.s., but had different topographies, Task $\times$ Recording Site $F_{6,66}=3.7, p=.01$, with positivity spreading more to anterior sites in the oddball go/no-go than in the combination go/no-go task.

\subsubsection{S2-evoked negativity}

The oddball effect on response selection, reflected in S2evoked negativity, was largest at FCz (Recording Site: $F_{6,66}=7.3$, 
$p=.008$, Task $\times$ Recording Site, $F_{18,198}=1.4$, n.s.). Negativity differed between tasks (main effect of Task: $F_{3,33}=8.7, p=.001$ ) being largest in the combination task in pair-wise comparisons to each of the other three tasks, $F_{1,11} \geq 15.3, p \leq .002$, which did not differ from each other, $F_{1,11} \leq 0.5, p \geq .49$.

\subsubsection{Response times and error rates}

Mean RTs and error rates are displayed in the right column of Fig. 3.

4.3.3.1. Response times. Overall, RTs differed between tasks, $F_{3,33}=51.3, p<.001$, because responses were fastest in the oddball $\left(F_{1,11} \geq 51.3, p<.001\right.$ vs. each of the three other tasks), slowest in combination $\left(F_{1,11} \geq 28.0, p<.001 \mathrm{vs}\right.$. each of the three other tasks) and did not differ between the two go/no-go tasks $\left(F_{1,11}=0.6\right.$, n.s. $)$. Responses were slower after rare than after frequent $S 1\left(F_{1,11}=58.1\right.$, $p<.001)$. This effect differed between tasks, Frequency $\times$ Task, $F_{3,33}=24.8, p<.001$. To localize this effect, rare- minus frequent-S1 differences were formed for each task, and these oddball effects were compared against zero and against each other by $t$-tests. All effects differed from zero: $16 \mathrm{~ms}$ in the oddball task $(p=.03)$, $33 \mathrm{~ms}$ and $39 \mathrm{~ms}$ in the oddball go/no-go and combination go/nogo tasks ( $p=.004$ and $p<.001)$ and $174 \mathrm{~ms}$ in the combination task $(p<.001)$. These oddball effects were largest in the combination task ( $p \leq .001$ for comparisons to the three other tasks), and smallest in the oddball task ( $p \leq .007$ in comparisons to the combination and the combination go/no-go tasks) though not different from the oddball go/no-go task $(p=.13)$ which also did not differ from the combination go/no-go task $(p=.55)$.

4.3.3.2. Error rates. The main effect of Task was significant, $F_{3,33}=16.3, p=.001$, because most errors occurred in the combination task $\left(F_{1,11} \geq 16.6, p \leq .002\right.$ vs. each of the three other tasks) while the three other tasks did not differ from each other $\left(F_{1,11} \leq 3.6, p \geq .09\right)$. More errors occurred after rare than after frequent $\mathrm{S} 1$ in the combination task, as indicated by effects of Frequency, $F_{1,11}=14.0, p=.003$, and of Frequency $\times$ Task, $F_{3,33}=16.3$, $p=.001$, with the rare- minus frequent-S1 differences differing from zero in the combination task only $(15 \%, p=.002)$ being larger in this task than in each of the other three tasks $(p \leq .002)$ which differed neither from zero $(p \geq .08)$ nor from each other $(p \geq .10)$.

False alarm rates in no-go trials (gray dashed lines in Fig. 3) occurred more often after frequent than after rare $S 1, F_{1,11}=10.6$, $p=.008$, without difference between the two tasks, $F_{3,33}<1.6$, $p>.23$.

\subsection{Discussion}

Experiment 3 was successful in obtaining an oddball effect that lay in-between the large oddball effect in the standard oddball task and the small effect in the combination task. This result will be addressed in Section 5.

Besides, the two versions of the go/no-go task were found to differ in the contributions of anterior recording sites to the topographies of their P3 oddball effects. One might assume that this effect was related to the usual finding of more anterior topographies of P3s evoked by no-go than by go stimuli (e.g., Pfefferbaum, Ford, Weller, \& Kopell, 1985; Verleger, Paehge, Kolev, Yordanova, \& Jaśkowski, 2006). But the rare S1 was not a no-go stimulus, because the go/no-go decision was triggered by S2. Moreover, the large difference in amplitudes between the two go/no-go tasks on the one hand and the oddball task on the other hand made it difficult to determine which one of the two go/no-go tasks resembled more the standard topography as obtained in the oddball task (Urbach \& Kutas, 2006). So it remains unclear what was the reason of these changed topographies. Perhaps these subtle differences were not in response to $\mathrm{S} 1$ but were due to slight differences between tasks in preparing for perceiving S2 and for executing the following response, similar to the task differences in pre-stimulus potentials described by Berchicci, Lucci, Pesce, Spinelli, and Di Russo (2012).

\section{General discussion}

\subsection{Summary of results}

We had previously reported that the effect of stimulus infrequency on the P3 potential ("oddball effect") evoked by S1 in a sequence of two stimuli ( $\mathrm{S} 1$ and $\mathrm{S} 2$ ) is much reduced when responses to this combination of stimuli are difficult to determine (Verleger, Baur, et al., 2014). The present study aimed at elucidating that previous finding, asking the question what aspect of that previous task was responsible for this reduction.

Experiment 1 tested the hypothesis that the decisive factor was the lack of associations from S1 to responses. But oddball effects were as reduced in the "combination 80\%" task which allowed for such associations as in the original "combination 50\%" task.

Experiment 2 tested the hypothesis that the decisive factor is given by complexity of S-R mapping. But less complex stimulus-response mappings led to equally large reductions of the oddball effect on P3b. This result was obtained although these tasks were indeed less difficult than the original combination task, as indicated by faster response times, less errors, and decreased S2evoked negativity.

Experiment 3 tested the hypothesis that the decisive factor is increased S2 relevance. Indeed, when the two S2 alternatives served as "go" or "no-go" signals, thereby attaining intermediate relevance between indiscriminately serving as "go" signals (in the oddball task) and being indispensable in determining the response (in the combination task), the oddball effect on S1-evoked P3b was intermediate between the large effect in the oddball task and the small effect in the combination task.

Thus, it appears that the oddball effect on S1-evoked P3 is related to the importance of $\mathrm{S} 1$ relative to $\mathrm{S} 2$ in defining the responses: The effect is large when responses are completely determined by $\mathrm{S} 1$ but becomes smaller and smaller the more relevance is attained by $\mathrm{S} 2$. This happened even though absolute relevance of S1 always remained the same: In all tasks, information provided by $\mathrm{S} 1$ was essential for selecting the response.

\subsection{Account in terms of $S-R$ link hypothesis}

We have proposed (Verleger, Metzner, et al., 2014; Verleger, Baur, et al., 2014) that P3b indicates activation of established, but presently inactive S-R links. We argued that in the oddball task, the frequent link (e.g., "X-left") does not need much reactivating, due to its being anyway activated most of the time. In contrast, the rare link (e.g., "U-right") is not active most of the time, therefore needs reactivating when the rare stimulus is perceived, which is indicated by P3b.

In the present version of the oddball task (Experiments 1 and 3) participants had to wait for the "go" signal (S2). But since the "go" signal inadvertently appeared, without any variation, activating these links made sense already when perceiving S1. We assume that this is the reason why the large oddball effect was evoked by S1, similarly though less distinct as when there would have been no extra "go" signal (cf. direct vs. delayed reaction in Johnson, Barnhardt, \& Zhu, 2003; Kok \& Looren De Jong, 1980; 0 ms vs. 400 ms SOA in Verleger, Metzner, et al., 2014). 
In go/no-go tasks, with no response required by $\mathrm{S} 2$ in the no-go case, S-R links may be only pre-activated by S1 to reach some level of intermediate activation, to be further activated by an S2 "go" stimulus or to be deactivated by an S2 "no-go" stimulus (Verleger et al., 2006). This scenario fits our results in the go/no-go tasks where P3 evoked by the frequent S1 is virtually non-existent (because this frequently used link is sufficiently preactivated) whereas P3 evoked by rare S1 is of intermediate size (being only pre-activated, rather than fully activated as in the oddball task) and where both frequent and rare S1 are followed by appreciable and equally large S2-evoked P3s (because the difference from pre-activation to full activation is equal for both $S-R$ links).

In the combination task, again S-R links might be pre-activated by $\mathrm{S} 1$ already. However, different from the go/no-go task, not one definite S-R link can be pre-activated after seeing S1 but rather two of them, e.g., "X \& blue - left", "X \& yellow - right". This might still work for the frequent $\mathrm{S} 1$, but may approach capacity limits for the rarely used links with rare S1, because holding four S-R links in working memory might be too demanding. This might be the reason why P3 evoked by infrequent S1 is smaller than in the go/no-go task. In the same vein, one of the two pre-activated frequent $S-R$ links in the combination task might be easily fully activated in response to S2, indicated by the S2-evoked P3 being as large after frequent $\mathrm{S} 1$ in the combination task as in the go/no-go task. In contrast, by not being pre-activated, the two infrequent S-R links cannot be simply activated by perceiving S2 but have to be newly assembled, indicated by the S2-evoked negativity which is the larger the more difficult is this assembly.

All these detailed considerations are post hoc but may serve to demonstrate that $\mathrm{S}-\mathrm{R}$ link hypothesis of P3b may cover these results. Alternative accounts of $\mathrm{P} 3 \mathrm{~b}$, in terms of working-memory ("context") updating (Donchin \& Coles, 1988), consciousness (Dehaene, Sergent, \& Changeux, 2003) or decision-making (Kelly \& O'Connell, 2013) and response monitoring (Gajewski \& Falkenstein, 2013; Verleger et al., 2005) would as well need specifications to account for these data. For example, we do not see how an account in terms of consciousness can be applied to the simple oddball effect at all: Even though greatly differing in P3 amplitudes, frequent and rare stimuli do not differ in terms of conscious perception. Nor are the rare S1 letters perceived less consciously in the combination task than in the oddball task, but do evoke a much smaller P3. The context updating hypothesis, on the other hand, can certainly account for the oddball effect (cf. Verleger, 1988, for an in-depth analysis of the hypothesis). It is not immediately obvious, though, how this hypothesis may explain the basic phenomenon studied in this paper which was the reduction of the oddball effect in the combination task compared to the oddball task. An additional assumption within the framework of this hypothesis might argue with the relative relevance of $\mathrm{S} 1$ and $\mathrm{S} 2$. But a problem in this respect is that this hypothesis has to cope with its corollary that P3 is affected by "stimulus evaluation" only (reiterated recently by Kamp, Brumback, \& Donchin, 2013). By excluding processes involved in selecting responses, this stipulation makes it quite difficult to account for the positive result achieved in Experiment 3, which we described as indicating the importance of S1 relative to $\mathrm{S} 2$ in defining the responses. Similarly to the contextupdating hypothesis, decision-making and response-monitoring hypotheses may easily account for the oddball effect, by stating that easy decisions which do not need much monitoring (with rare oddball stimuli) are accompanied by larger P3s than simple perseverations (with frequent stimuli) which do not need any decision and monitoring. Yet these hypotheses meet with the difficulty that truly hard decisions where much monitoring is needed were not accompanied by P3s in the present data, let alone by further increased P3s, but rather by the anterior S2-evoked negativity as measured in all three experiments in the combination task. Thus, it appears that all these hypotheses need further refinement as well.

\section{Acknowledgment}

This work was supported by grant VE110/17-1 from Deutsche Forschungsgemeinschaft to RV.

\section{References}

Berchicci, M., Lucci, G., Pesce, C., Spinelli, D., \& Di Russo, F. (2012). Prefrontal hyper activity in older people during motor planning. Neurolmage, 62, 1750-1760.

Callaway, E. (1983). The pharmacology of human information processing. Psychophysiology, 20, 359-370.

Dehaene, S., Sergent, C., \& Changeux, J. P. (2003). A neuronal network model linking subjective reports and objective physiological data during conscious perception. Proceedings of the National Academy of Science of the United States of America, 100, 8520-8525

Donchin, E., \& Coles, M. G. H. (1988). Is the P300 component a manifestation of context updating? Behavioral and Brain Sciences, 11, 357-374.

Duncan, C. C., Barry, R. J., Connolly, J. F., Fischer, C., Michie, P. T., Näätänen, R., et al. (2009). Event-related potentials in clinical research: Guidelines for eliciting, recording, and quantifying mismatch negativity, P300, and N400. Clinical Neurophysiology, 120, 1883-1908.

Duncan-Johnson, C. C., \& Donchin, E. (1977). On quantifying surprise: The variation of event-related potentials with subjective probability. Psychophysiology, $14,456-467$.

Gajewski, P. D., \& Falkenstein, M. (2013). Effects of task complexity on ERP components in Go/Nogo tasks. International Journal of Psychophysiology, 87, 273-278.

Gerson, A. D., Parra, L. C., \& Sajda, P. (2005). Cortical origins of response time variability during rapid discrimination of visual objects. NeuroImage, 28, 342-353.

Hanslmayr, S., Pastötter, B., Bäuml, K.-H., Gruber, S., Wimber, M., \& Klimesch, W. (2008). The electrophysiological dynamics of interference during the Stroop task. Journal of Cognitive Neuroscience, 20, 215-225.

Johnson, R., Jr., \& Donchin, E. (1980). P300 and stimulus categorization: Two plus one is not so different from one plus one. Psychophysiology, 17, 167-178.

Johnson, R., Jr., Barnhardt, J., \& Zhu, J. (2003). The contribution of executive processes to deceptive responding. Neuropsychologia, 42, 878-901.

Johnson, R., Jr., Henkell, H., Simon, E., \& Zhu, J. (2008). The self in conflict: The role of executive processes during truthful and deceptive responses about attitudes. NeuroImage, 39, 469-482

Kamp, S.-M., Brumback, T., \& Donchin, E. (2013). The component structure of ERP subsequent memory effects in the Von Restorff paradigm and the word frequency effect in recall. Psychophysiology, 50, 1079-1093.

Kelly, S. P., \& O'Connell, R. G. (2013). Internal and external influences on the rate of sensory evidence accumulation in the human brain. The Journal of Neuroscience, 33, 19434-19441.

Kok, A., \& Looren De Jong, H. (1980). Components of the event-related potential following degraded and undegraded visual stimuli. Biological Psychology, 11, $117-133$.

Kotchoubey, B. (2014). First love does not die: A sustaining primacy effect on ERP components in an oddball paradigm. Brain Research, 1556, 38-45.

Lang, W., Obrig, H., Lindinger, G., Cheyne, D., \& Deecke, L. (1990). Supplementary motor area activation while tapping bimanually different rhythms in musicians. Experimental Brain Research, 79, 504-514.

McCarthy, G., \& Wood, C. C. (1985). Scalp distributions of event-related potentials: An ambiguity associated with analysis of variance models. Electroencephalography and Clinical Neurophysiology, 62, 203-208.

O'Connell, R. G., Dockree, P. M., \& Kelly, S. P. (2012). A supramodal accumulationto-bound signal that determines perceptual decisions in humans. Nature Neuroscience, 15, 1729-1735.

Oldfield, R. C. (1971). The assessment and analysis of handedness: The Edinburgh Inventory. Neuropsychologia, 9, 97-113.

Ouyang, G., Herzmann, G., Zhou, C., \& Sommer, W. (2011). Residue Iteration Decomposition (RIDE): A new method to separate ERP components on the basis of latency variability in single trials. Psychophysiology, 48, 1631-1647.

Pfefferbaum, A., Ford, J. M., Weller, B. J., \& Kopell, B. S. (1985). ERPs to response production and inhibition. Electroencephalography and Clinical Neurophysiology, $60,423-434$

Poli, R., Cinel, C., Citi, L., \& Sepulveda, F. (2010). Reaction-time binning: A simple method for increasing the resolving power of ERP averages. Psychophysiology. 47, 467-485.

Ritter, W., \& Vaughan, H. G., Jr. (1969). Averaged evoked responses in vigilance and discrimination: A reassessment. Science, 164, 326-328.

Rosenbaum, D. A. (1980). Human movement initiation: Specification of arm, direction, and extent. Journal of Experimental Psychology: General, 109, 444-474.

Saville, C. W. N., Dean, R. O., Daley, D., Intrilligator, J., Boehm, S., Feige, B., et al. (2011). Electrocortical correlates of intra-subject variability in reaction times: Average and single-trial analyses. Biological Psychology, 87, 74-83.

Squires, N. K., Squires, K. C., \& Hillyard, S. A. (1975). Two varieties of long-latency positive waves evoked by unpredictable auditory stimuli in man. Electroencephalography and Clinical Neurophysiology, 38, 387-401. 
Urbach, T. P., \& Kutas, M. (2006). Interpreting event-related brain potential (ERP) distributions: Implications of baseline potentials and variability with application to amplitude normalization by vector scaling. Biological Psychology, 72, 333-343.

Verleger, R. (1988). Event-related potentials and cognition: A critique of the context updating hypothesis and an alternative interpretation of P3. Behavioral and Brain Sciences, 11, 343-356.

Verleger, R., Jaśkowski, P., \& Wascher, E. (2005). Evidence for an integrative role of P3b in linking reaction to perception. Journal of Psychophysiology, 19, 165-181.

Verleger, R., Paehge, T., Kolev, V., Yordanova, J., \& Jaśkowski, P. (2006). On the relation of movement-related potentials to the go/no-go effect on P3. Biological Psychology, 73, 298-313.
Verleger, R., Schroll, H., \& Hamker, F. (2013). The unstable bridge from stimulus processing to correct responding in Parkinson's disease. Neuropsychologia, 51 2512-2525.

Verleger, R., Metzner, M. F., Ouyang, G., Śmigasiewicz, K., \& Zhou, C. (2014). Testing the stimulus-to-response bridging function of the oddball-P3 by delayed response signals and residue iteration decomposition (RIDE). NeuroImage, 100 271-280.

Verleger, R., Baur, N., Metzner, M. F., \& Śmigasiewicz, K. (2014). The hard oddball: Effects of difficult response selection on stimulus-related P3 and on responserelated negative potentials. Psychophysiology, 51, 1089-1100. 